\title{
Mecanismo de Inferencia Sensible al Contexto para la Integración de Procesos de Negocio en Acreditación Académica
}

\author{
Jorge E. Giraldo(1,2), Demetrio A. Ovalle(1) y Flavia M. Santoro(3) \\ (1) Universidad Nacional de Colombia, Facultad de Minas, Departamento de Ciencias de la Computación \\ y la Decisión, Grupo de Investigación GIDIA, Medellín-Colombia (e-mail: jegiraldp@unal.edu.co, \\ dovalle@unal.edu.co) \\ (2) Politécnico Colombiano Jaime Isaza Cadavid Facultad de Ingeniería, Medellín-Colombia \\ (e-mail: jegiraldo@elpoli.edu.co) \\ (3) Universidade Federal do Estado do Rio de Janeiro, Brasil (e-mail: flavia.santoro@uniriotec.br)
}

Recibido Nov. 17, 2015; Aceptado Ene. 22, 2016; Versión final Feb. 19, 2016, Publicado Ago. 2016

\begin{abstract}
Resumen
El objetivo de este artículo es proponer un mecanismo de inferencia para la integración de procesos de negocio dependiente de información contextual asociada al dominio de ejecución. La metodología utilizada por el mecanismo de inferencia propuesto se compone de un modelo de contexto, formalizado a través de situaciones contextuales basadas en restricciones de tipo organizacional y operacional, y un algoritmo, que tienen como función principal comparar semántica y sintácticamente las variables con el fin de determinar su inclusión o no en la integración. La validación del mecanismo se realizó sobre el caso de estudio del proceso de acreditación académica. Después de aplicar el mecanismo de inferencia propuesto se puede concluir que se mejora la selección de las variantes a integrar, teniendo en cuenta la información contextual. Esto redunda en la correcta gestión de la información del proceso tal como recolección y almacenamiento, entre otros.
\end{abstract}

Palabras clave: integración de procesos de negocio; modelo sensible al contexto; algoritmo de inferencia; situaciones contextuales; acreditación académica

\section{Context-Aware Inference Mechanism for Business Process Integration in Academic Accreditation}

\begin{abstract}
The aim of this paper is to propose an inference mechanism for business process integration based on contextual information associated with the execution domain. The methodology used by the proposed inference mechanism is composed of a context model, formalized through contextual situations based on organizational and operational constraints, and an algorithm, which have as main function to semantic and syntactically compare variables to determine their inclusion or not in the integration. The validation of the mechanism was performed using as case study the academic accreditation process. After applying the proposed inference mechanism it can be concluded that the selection of variants is improved for process integration, taking into account the contextual information. This helps in improving the correct management of the process information such as gathering and storage, among others.
\end{abstract}

Keywords: business process integration; context-aware model; inference algorithm; contextual situations 


\section{INTRODUCCIÓN}

Un proceso de negocio se conoce como la cadena de eventos, actividades y decisiones que al final dan valor agregado a una organización y a sus clientes. La forma en que los procesos son diseñados y ejecutados, afecta tanto la percepción que los clientes tienen sobre la calidad del servicio como la eficiencia de los mismos (Dumas et al., 2013). El modelado y representación de los procesos de negocio abarca una gran variedad de aplicaciones, entre ellas se destacan cadenas de suministro de recursos (Sanchis et al., 2009), gestión de pedidos en ambientes soportados por tecnologías web (Carrillo y Franky, 2014), así como la simulación de procesos de negocio como soporte didáctico de aprendizaje (Giraldo y Pinilla, 2015).

La gestión de calidad en instituciones de educación superior es un procedimiento constante que busca el mejoramiento continuo en aspectos relacionados con la docencia, investigación y extensión. De esta manera se asegura el diseño apropiado de instrumentos de evaluación y seguimiento (Llanera et al., 2014), teniendo en cuenta aspectos inherentes a la gestión de los procesos de planificación, implementación y ejecución de cursos presenciales $\mathrm{y} / \mathrm{o}$ virtuales.

La integración de procesos de negocio (BPI - Por sus siglas en inglés), tiene como objetivo obtener un proceso de negocio a partir de sus variantes, por medio de la fusión de varios modelos en uno solo. De esta manera se busca que el resultado de la integración de cierto modo entre a reemplazar los procesos anteriores (Morrison et al., 2009). Según Dey (2001), se define el contexto como "cualquier información que puede ser usada para caracterizar la situación de una entidad. Una entidad es una persona, lugar u objeto que se considera relevante en la interacción entre el usuario y la aplicación, incluyendo al usuario y la aplicación misma". Es decir, se realiza un sensado al ambiente haciendo uso de razonamiento de la información del contexto para posteriormente caracterizar las situaciones y con ellas tomar acciones específicas.

En el área de procesos de negocio, existen trabajos de investigación que tienen en cuenta la sensibilidad al contexto principalmente para: (1) la gestión de roles y sus competencias (2) control en el dominio de ejecución de las variantes (3) ejecución actividades y flujos de información. Tavares et al. (2011) presentan una arquitectura para el manejo de la flexibilidad de los procesos de negocio teniendo en cuenta información contextual recolectada del ambiente y que a su vez puede ser controlada en tiempo real. Por su parte Mattos et al. (2012) proponen una aproximación formal para el contexto en procesos de negocio basada en modelos conceptuales. Dicha formalización busca identificar la situación de una actividad con el fin de soportar la toma de decisiones durante la ejecución de un proceso. Li et. al. (2011) presentan una solución a la administración de colecciones de variantes de procesos, a partir de la validación de su similitud, por medio de su presentación en redes de restricciones de procesos. De esta forma las restricciones son clasificadas en: estratégicas, operacionales, regulatorias y contractuales.

Una variante se considera como el cambio de la estructura de un proceso de negocio, específicamente en las instancias de un modelo de referencia. Las variantes de los procesos se consideran como descripciones "similares pero diferentes" (Döhring et al., 2014), ya que poseen características en común y características que las hacen diferentes. A medida que las variantes de los procesos aumentan se deben diseñar repositorios para su almacenamiento, recuperación y consulta (Reichert et. al, 2014). Esta tendencia está motivada por el diseño continuo de variantes a partir de modelos de referencia (Hallerbach, 2010) y la consolidación en un solo proceso a partir de un conjunto de dichas variantes (La Rosa et al., 2013). Una vez se realiza una actualización en el modelo de referencia es necesario propagar los cambios a las variantes asociadas. La administración de las variantes puede realizarse de manera individual o por medio del agrupamiento de las variantes en un solo proceso (Lohrmann y Reichert, 2012). Sin embargo, no todas las variantes pueden soportar la propagación de los cambios, debido a que su ejecución puede depender de un contexto específico (Ploesser et al., 2009).

En importante resaltar el papel que desempeña de la información contextual en la ejecución de los procesos de negocio y cómo el dominio desempeña un papel importante en la definición de dependencias contextuales. Por lo anterior es importante ahondar en el diseño de mecanismos de decisión que determine el conjunto de variantes a integrar, donde no se afecte su ejecución a partir de una actualización hecha. Adicionalmente, el dominio y su relación con los procesos, convoca a definir estrategias de tipo semántico que faciliten la su valoración dentro de una situación propia de un contexto de ejecución.

El objetivo de este artículo es presentar el diseño de un mecanismo de inferencia sensible al contexto para la integración de procesos académicos en acreditación académica, soportado por los siguientes elementos: (1) modelo de contexto formalizado a través de situaciones contextuales basadas en restricciones de tipo organizacional y operacional y (2) una propuesta de algoritmo de inferencia basado en análisis de información situacional y su dependencia con el dominio de ejecución. 


\section{ACREDITACIÓN ACADÉMICA}

El Sistema Nacional de Acreditación ha sido propuesto con el fin de conservar y velar por la calidad académica en los procesos académicos universitarios. De esta manera, por medio de la acreditación se logra un reconocimiento por parte del estado Colombiano, hacia las instituciones de educación superior y sus programas académicos, fomentando una dinámica de mejoramiento continuo orientado hacia el establecimiento de una cultura de la calidad y de sistemas consolidados de autoevaluación. "El proceso de acreditación tiene un carácter voluntario y canaliza los esfuerzos de las instituciones para llevar a cabo la evaluación sistemática de sus programas y, en general, del servicio que prestan a la sociedad." (CNA, 2013).

La acreditación se divide en dos grandes procesos, estos son: (a) Registro Calificado de condiciones mínimas de calidad y (b) acreditación en alta calidad, la cual busca ser una actividad constante para el mejoramiento continuo. En la Figura 1, se presenta la aproximación metodológica propuesta por CNA (2013), la cual consta de cinco (5) fases, a seguir:

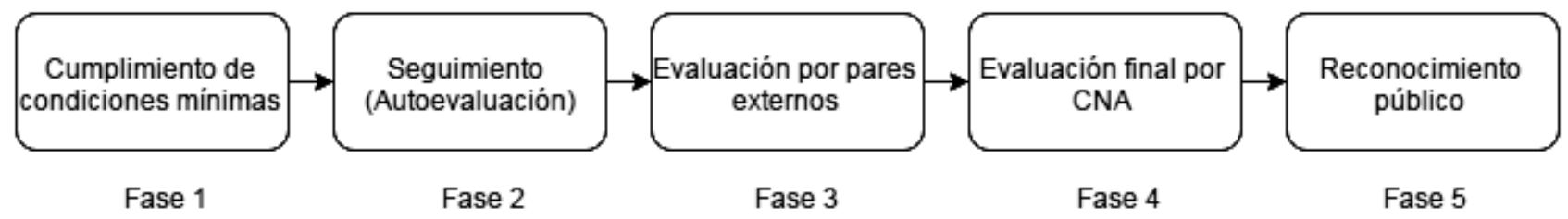

Fig. 1. Aproximación metodológica para la acreditación académica (CNA, 2013).

Fase 1 - Cumplimiento de condiciones mínimas: Se basa en las condiciones aceptables para que cualquier programa de educación superior pueda ofertarse a la comunidad académica. Se considera un tipo de licencia de funcionamiento otorgada por el Ministerio de Educación Nacional.

Las condiciones mínimas de funcionamiento se basan en los siguientes factores críticos de éxito:

Factor 1: Misión y visión de la institución y del programa

Factor 2: Estudiantes

Factor 3: Profesores

Factor 4: Formación en investigación

Factor 5: Visibilidad nacional e internacional

Factor 6: Procesos académicos

Factor 7: Impacto de egresados en el medio

Factor 8: Bienestar institucional

Factor 9: Procesos administrativos

Factor 10: Recursos físicos y financieros

Es importante señalar que cada uno de los factores mencionados es susceptible a cambios generados por el entorno y por la dinámica propia de cada una de las instituciones donde se aplica, así como de cada uno de sus actores (e.g. estudiantes, docentes, empleados, empleadores, egresados, administrativos, directivos).

Fase 2 - Seguimiento por parte de la institución: La institución debe asumir el liderazgo del seguimiento de la acreditación de alta calidad, denominada "autoevaluación académica". Parte del proceso consiste en el cambio de actitud y conciencia de la comunidad educativa.

Básicamente el seguimiento consiste en: Sensibilización, levantamiento de información, análisis de resultados, planeación y ejecución acciones de mejoramiento en el corto, mediano y largo plazo. Se debe tener en cuenta hacer seguimiento sistemático a dichas mejoras.

Como parte de los lineamientos sugeridos por el estado colombiano, se sugiere que dicho seguimiento se haga por lo menos cada 2 años. Sin embargo, este periodo de tiempo depende en gran medida de las dinámicas propias de cada institución o región.

Fase 3 - Evaluación por parte de pares externos: Una vez la institución considera cumplir con las condiciones para la acreditación académica, el Ministerio de Educación asigna evaluadores (pares académicos) externos para su evaluación, la cual se realiza de forma presencial. Como resultado los pares académicos emiten un concepto basado en los criterios de calidad definidos. 
La visita de los evaluadores consiste en: reuniones con todos los miembros de la comunidad académica, con el fin de evidenciar lo expuesto en los informes de autoevaluación, visitas guiadas por los espacios y ambientes de aprendizaje de los programas académicos, emisión de un concepto inicial basado en acciones de fortalecimiento y mejoras, que constituyen requisitos para la recomendación ante el estado colombiano para el otorgamiento de la acreditación en alta calidad.

Fase 4 - Evaluación Final: A partir del concepto definido en la fase 3, el Consejo Nacional de Acreditación, realiza una evaluación final y determina si el programa académico analizado se considera acreditado. Esta fase puede tardar algunos meses, debido a la periodicidad en las reuniones de los expertos consejeros del Ministerio de Educación Nacional.

Fase 5 - Reconocimiento público: Como su nombre lo indica y cuando el programa es acreditado, esta fase considera la difusión del reconocimiento público otorgado al programa académico. El Ministerio de Educación Nacional informa por sus canales oficiales a la comunidad académica el logro obtenido.

Es importante señalar que el logro de la obtención de la acreditación en alta calidad de un programa académico le otorga beneficios tales como: derecho a presentarse a convocatorias de proyectos de investigación, acceso a recursos institucionales, derecho a abrir el mismo programa en otras sedes de la institución.

\section{DISEÑO DEL MECANISMO DE INFERENCIA PARA LA BPI}

El mecanismo de inferencia para la BPI está compuesto por: un modelo de contexto descrito a partir de situaciones y un algoritmo de inferencia, los cuales se presentan a continuación.

\section{Modelo de contexto}

El modelo del contexto está compuesto por situaciones sobre elementos contextuales con información asociada a restricciones. Un elemento contextual refiere a aquellos elementos que dependen directamente de la ejecución del proceso. Cada elemento contextual se compone de la entidad contextual y sus atributos. Los atributos contextuales están definidos al interior del dominio de ejecución por medio de etiquetas contextuales de dominio. De esta manera un dominio puede asociar sus elementos a varios contextos de acuerdo a las situaciones.

Las restricciones de un proceso de negocio dependen directamente de la información asociada, esta puede ser de tipo organizacional u operacional (Schubert y Legner, 2011). Las primeras dependen directamente de la lógica de negocio y su organización; por su parte las restricciones operacionales, se relacionan con la estructura del proceso, específicamente con sus bloques de construcción (actividades, gateways).

Una restricción organizacional se enfoca a delimitar los valores que puede tomar el atributo de un elemento contextual, con base en su relación con un dominio. Si su valor se encuentra asociado a un Elemento de Dominio Contextual, entonces el elemento contextual tiene importancia contextual para la integración. Una restricción operacional se enfoca a delimitar los valores un Elemento de Dominio Contextual. Si la actividad asociada a dicho elemento de dominio posee valores dentro del mismo rango, se considera que tiene importancia contextual.

Con base en el trabajo realizado en (Carvalho et. al, 2015), se propone las representación de las siguientes situaciones basadas en restricciones, que definen el modelo de contexto para la integración de procesos.

Situación 1 - Dado un evento de adición - Ev(add) - sobre un Elemento de Dominio Contextual -eCtx-y se satisface una restricción organizacional - $R_{0 r g}$ - sobre un Concepto de dominio - $C d$-, entonces se presenta una situación que tiene prioridad contextual

$$
S t(E v(a d d), e C t x) \wedge R_{O r g}(C d) \equiv p C t x
$$

Situación2 - Dado un evento de adición - Ev(del) - sobre un Elemento de Dominio Contextual -eCtx-y se satisface una restricción organizacional - $R_{\text {org- }}$ sobre un Concepto de dominio - $C d-$, entonces se presenta una situación que tiene prioridad contextual.

$$
S t(E v(d e l), e C t x) \wedge R_{O r g}(C d) \equiv p C t x
$$


Situación 3 - Dado un evento de adición - Ev(add) - sobre un Elemento de Dominio Contextual -eCtx-y se satisface una restricción operacional - $R_{O p}$ - sobre un Concepto de dominio - $E d$-, entonces se presenta una situación que tiene prioridad contextual.

$$
S t(E v(a d d), e C t x) \wedge R_{O p}(C d) \equiv p C t x
$$

Situación 4 - Dado un evento de eliminación - Ev(del) - sobre un Elemento de Dominio Contextual -eCtx- y se satisface una restricción operacional - $R_{O p}$ - sobre un Concepto de dominio - $E d$-, entonces se presenta una situación que tiene prioridad contextual.

$$
S t(E v(d e l), e C t x) \wedge R_{O p}(C d) \equiv p C t x
$$

La importancia de identificar situaciones que pueden afectar el funcionamiento de los procesos luego de la propagación de cambios, puede evitar que se tomen decisiones y/o se asignen recursos innecesarios para la ejecución de algunas instancias, ya sea por afectaciones directas sobre los resultados o sobre la información que se manipula.

\section{Algoritmo de inferencia}

El algoritmo propuesto busca determinar el grado de similitud entre un modelo de referencia y sus variantes. Para ello se aplican dos métricas para la comparación de modelos de procesos de negocio, estas son: métrica de similitud semántica y métrica de similitud estructural (Becker y Laue, 2012).

La similitud semántica consiste en comparar entre 2 procesos, la pertenencia conceptual de cada una de las actividades a un dominio, así como la determinar los eventos generados para la obtención de dicha variante, es decir, si se realizó un evento de eliminación o de adición. Para ello se hace uso de una ontología de variantes de proceso que facilita el razonamiento.

Por su parte la similitud sintáctica consiste en comparar la estructura de los procesos, determinando diferencias en los elementos que la componen, para ello se tiene en cuenta: número de actividades, participantes, reglas y transiciones.

Una vez se obtienes las medidas individuales se procede a comparar los procesos desde el punto de vista semántico y sintáctico, teniendo como medida el grado de similitud. Sus valores son probabilísticos, siendo una probabilidad de 1.0 cuando los procesos son iguales y 0 cuando no tienen ningún grado de similitud.

Con base en lo anterior el algoritmo de inferencia tiene los siguientes insumos de información:

i) Modelo de referencia: Proceso de negocio abstracto que sirve para la definición de variantes.

ii) Repositorio de variantes de evolución y petición: Conjunto de procesos descritos en lenguaje XPDL. Su representación es de tipo objetual con una estructura tipo árbol.

iii) Modelo de contexto: Archivo XML que describe las situaciones y las marcas contextuales asociadas el dominio.

iv) Objetivo de integración: Consiste en un nuevo modelo de proceso, para lo que el algoritmo debe identificar los cambios con respecto al modelo de referencia actual

El algoritmo manipula como objetos a los procesos, con el fin de facilitar su caracterización en términos de las métricas para posteriormente aplicar el análisis contextual. El resultado del algoritmo es la clasificación de las variantes en 2 grupos, aquellas recomendadas para la integración y posterior propagación de cambios y el grupo de las variantes que debido a su grado de similitud no lo son.

El pseudocódigo se presenta en la Figura 2. Posterior a la generación de grupos de variantes, se procede a aplicar un "Filtro contextual", que permite identificar posibles situaciones que activen las restricciones y por ende realizar un análisis de las prioridades contextuales, lo que constituye en pieza clave para la generación del ranking.

\section{VALIDACIÓN DEL MECANISMO}

Se llevó a cabo la validación del mecanismo de inferencia a partir del proceso de autoevaluación académica descrito en la Figura 3, se ilustra el proceso de autoevaluación definido por la Institución Universitaria Politécnico Colombiano Jaime Isaza Cadavid, para que sea aplicado en cada uno de sus programas académicos. 


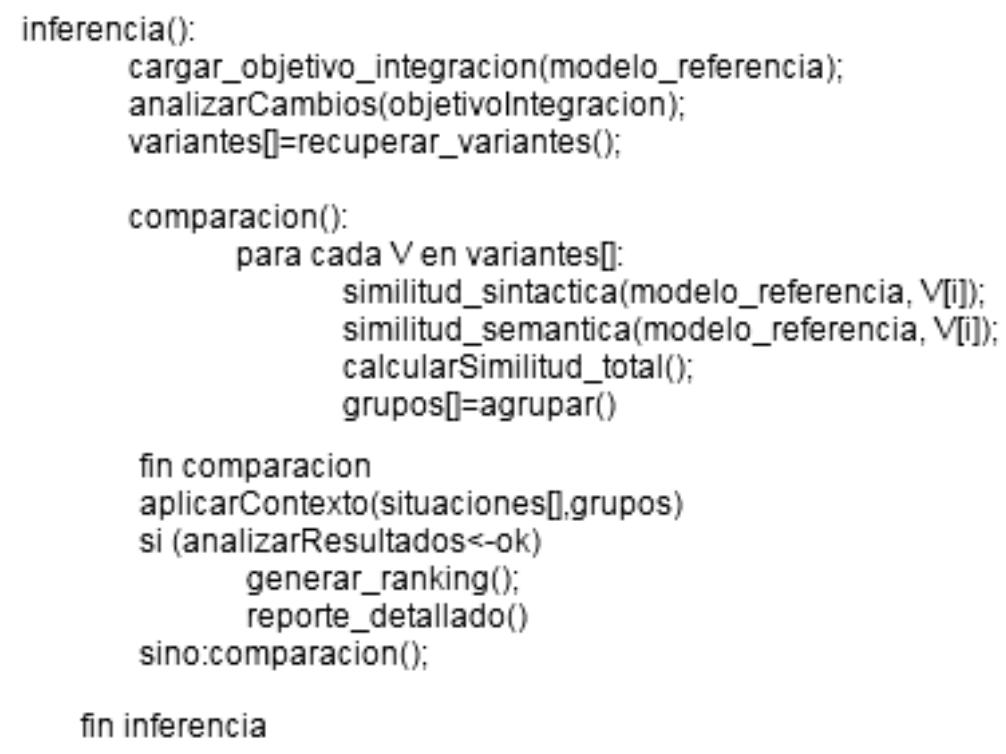

Fig. 2. Algoritmo de mecanismo de inferencia. Autoría Propia.

El Politécnico Colombiano cuenta con un total de 12 programas universitarios (duración 5 años), 20 programas Tecnológicos (duración 3 años) y 3 programas de tipo Técnico Profesional (duración 2 años), repartidos en 3 sedes, cada una en una ciudad del territorio Colombiano.

Se llevó a cabo la validación del mecanismo de inferencia a partir del proceso de autoevaluación académica descrito en la Figura 4, se creó un grupo de variantes distribuidas de la siguiente manera: i) 32 variantes por evolución propias del proceso; y ii) 54 variantes por petición generadas por los usuarios.

Cabe señalar que para cada proceso puede existir una o varias variantes, ya que la dichas instancias pueden ser creadas en distintos hitos en el tiempo, así como en distintas administraciones, lo que conlleva a depender de reglamentaciones gubernamentales y momentos históricos. Para las variantes por evolución se realizó un evento por eliminación de actividad, que afecta directamente a la lógica de negocio, mientras para las otras se propuso un evento de adición de actividad.

En la Figura 4 se presenta la plantilla de representación de un objetivo de integración. Los objetivos son descompuestos en colecciones refinadas de sub-objetivos y mediante el uso de ontologías de dominio se alinean con tareas específicas. Los objetivos indican las actividades que deben realizarse, cada proceso tiene un solo objetivo principal (Goal) y varios objetivos temporales (Temporal Goals). Un objetivo principal indica la actividad que finaliza el proceso y es aquella a la cual se desea llegar, mientras los objetivos temporales son aquellos transitorios.

Cada objetivo, sea principal o temporal, está compuesto por una o varias actividades. Cada actividad se asocia con una de las restricciones definidas y se compone de eventos externos o internos. Los eventos se asocian directamente con los niveles arquitectónicos de integración y con los escenarios.

Pueden existir eventos que afecten la lógica del proceso, los datos o la interfaz de visualización, según sea el caso. El bloque de declaración de restricciones se relaciona con los escenarios de integración, para los cuales se definen los siguientes elementos: (1) roles con sus habilidades para el escenario organizacional, (2) datos y su formato de codificación para el escenario técnico y (3) leyes dadas en términos de reglas de negocio.

\section{RESULTADOS Y DISCUSIÓN}

Se definen 2 grupos así: El "Grupo_Ok" para aquellas variantes que se recomienda incluir la integración y posterior propagación de cambios, yel "Grupo_No_OK" para aquellas que no. Para la definición de los grupos se aplicó una técnica de agrupamiento K-Means para lo cual se definieron los siguientes parámetros de configuración:

Valor del Centro $=5$,

Distancia de centro $=0,3$

Valor óptimo 1.0. 


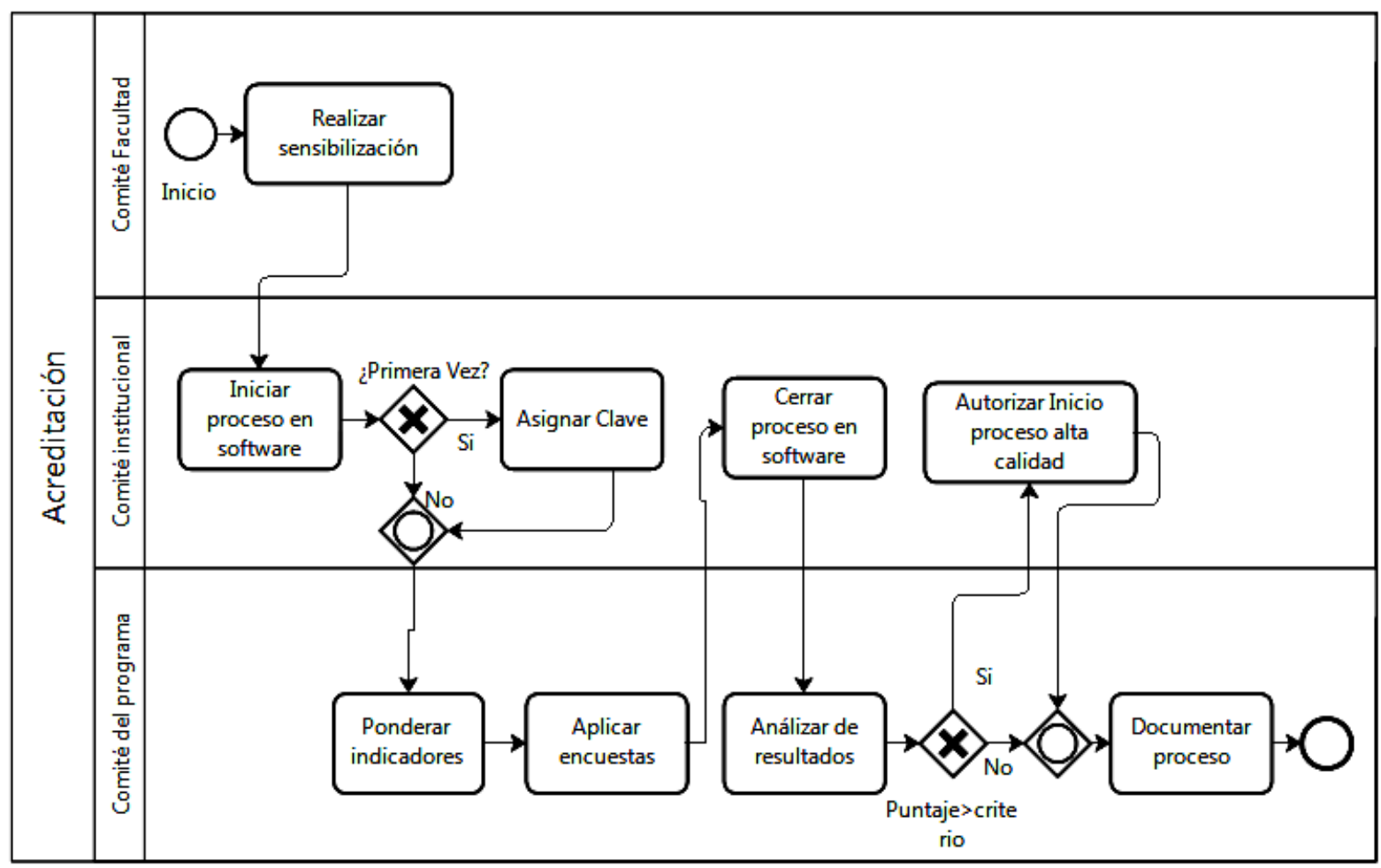

Fig. 3. Autoevaluación académica. Autoría Propia.

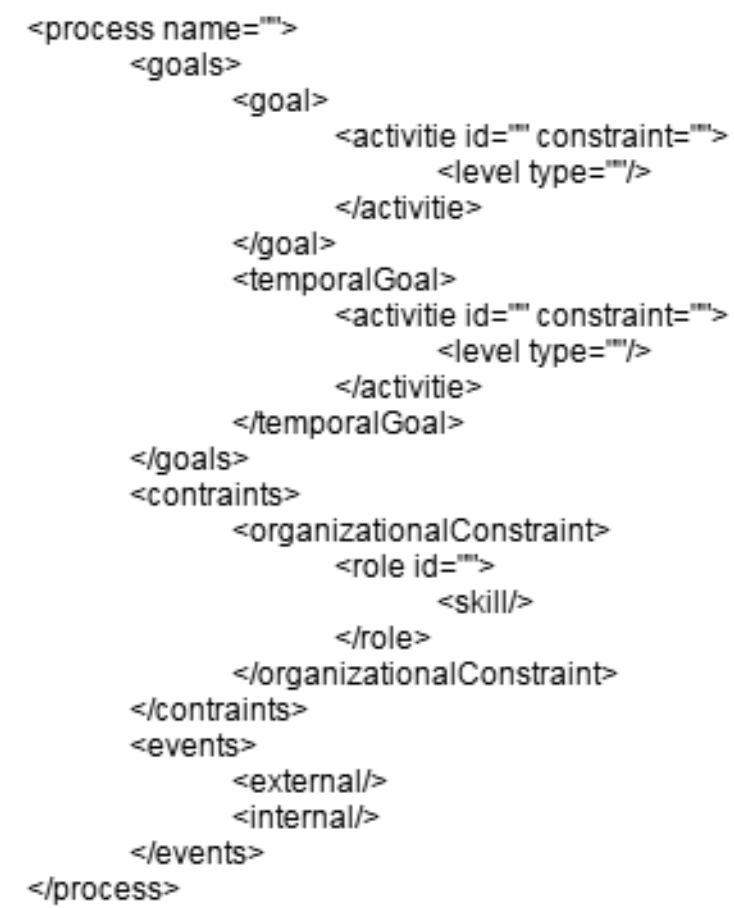

Fig. 4. Modelo de Proceso objetivo de integración. Autoría Propia.

Los valores de configuración se definen con base en la generación de 2 grupos, aquellos que están cerca del valor de centro y aquellos que no lo están. De esta manera aquellos que están más cerca al centro se consideran aptos para su inclusión en un procedimiento de integración; por su parte, aquellos que están lejos del centro se resaltan con alertas contextuales, advirtiendo al analista de procesos, que pueden ser susceptibles a fallas durante su ejecución.

Los resultados una vez realizados los procedimientos de comparación semántica y sintáctica son: Grupo_Ok=13 yGrupo_No_Ok=41. A partir de los datos obtenidos se aplicó el filtro contextual, basado en las siguientes situaciones, que según las restricciones definidas, tienen prioridad contextual alta: 
Situación 1:Hace referencia a que la actividad adicionada relacionada con el elemento contextual Institución (inst) y su atributo Seguimiento (seg) satisfacen la restricción organizacional R.

$$
\operatorname{St}(\operatorname{Ev}(\text { add }),(\text { inst }, \text { seg })) \wedge R_{\text {Org }}(\text { seg }) \equiv 1.0 \rightarrow \text { prioridad }
$$

Situación 2: Hace referencia a que la actividad eliminada se relaciona con un concepto del dominio y por ello su prioridad contextual puede variar.

$$
S t(E v(\text { del }),(\text { clave })) \wedge R_{O p}(\text { clave }) \equiv 1.0 \rightarrow \text { prioridad }
$$

Cabe señalar que al representar el contexto mediante situaciones, la comparación es susceptible a modificación en sus valores, lo que en términos de resultados significa que algunos elementos cambiarán de grupo de selección.

Con base en lo anterior, una vez aplicado el algoritmo de inferencia, los resultados obtenidos son:Grupo_Ok=5 y Grupo_No_Ok=49. Una vez se aplicó el filtro semántico al primer resultado del agrupamiento, 8 variantes cambiaron de grupo, indicando que no deben ser incluidas en el proceso de integración, ya que las situaciones tienen dependencia con el contexto y dominio de ejecución.

Es importante aclarar que el agrupamiento de variantes depende exclusivamente de las comparaciones realizadas contra su modelo de referencia, por lo anterior, si una variante es afectada por una situación específica, el mecanismo de inferencia simplemente recomienda no incluirlas.

\section{CONCLUSIONES}

El mecanismo propuesto y validado, permite demostrar que un razonamiento basado en situaciones contextuales, reduce el número de variantes susceptibles a la integración. De esta manera al propagar cambios desde un modelo de referencia es posible que las variantes no vean afectado su rendimiento en la ejecución.

El procedimiento de inferencia propuesto, al considerar factores de orden semántico, garantiza un soporte conceptual, evitando así el uso de actividades con igual nombre pero con diferente significado asociado a un dominio de ejecución. Por su parte, los factores estructurales (sintácticos) garantizan una mínima similitud a la hora de recuperación de las variantes.

En cuanto a la aplicación del mecanismo de inferencia, en el caso de estudio de acreditación académica, se puede concluir que es posible mejorar la selección de las variantes a integrar, teniendo en cuenta la información contextual asociada al dominio de ejecución. Lo anterior se materializa en una correcta gestión de la información involucrada en el proceso a la cual se aplican métodos de recolección y mecanismos de almacenamiento.

\section{AGRADECIMIENTOS}

La investigación presentada en este artículo está parcialmente financiada por el proyecto titulado: "Método de integración inteligente de procesos de negocio sensible y adaptado al contexto" de la Universidad Nacional de Colombia a través del programa de apoyos a posgrados, asociada al estudiante de doctorado Jorge $\mathrm{E}$. Giraldo Plaza con código Quipu 200000013723.

\section{REFERENCIAS}

Becker, M.y R. Laue, A comparative survey of business process similarity measures, Computers in Industry, Volume 63, Issue 2, Pages 148-167, ISSN 0166-3615 (2012)

Bucchiarone, A., B. Kessler, A. Marconi, M. Pistore y A.Sirbu, A Context-Aware Framework for Business Processes Evolution, Enterprise Distributed Object Computing Conference Workshops-EDOCW (2011)

Consejo Nacional de Acreditación - CNA, Lineamientos para la acreditación de programas de pregrado, Sistema Nacional de Acreditación. Bogota (2013). Disponible en línea en: http://www.cna.gov.co/1741/articles-186359_pregrado_2013.pdf(Visitado: Junio 2015)

Carrillo, M., C. Franky, Modelo SCLOUDPY para la gestión de pedidos en la nube, doi: 10.4067/S071807642014000400006. Información Tecnológica (en linea). volumen 25, número 4. La Serena-Chile (2014) 
Carvalho, J., F. Santoro y K.Revoredo, A method to infer the need to update situations in business process adaptation, Computers in Industry, Volume 71, Pages 128-143, ISSN 0166-3615 (2015)

Dey, A., Understanding and using context, Personal and Ubiquitous Computing, 5(1), 4-7 (2001)

Döhring, M.,H. Reijers, yS. Smirnov, Configuration vs. adaptation for business process variant maintenance: An empirical study, Information Systems, Volume 39, January (2014)

Giraldo, J., J. Pinilla, Simulación de procesos de negocio (BPSIM) como soporte didáctico en el aprendizaje de gestión de procesos de servicio, doi: 10.4067/S0718-50062016000100011.Revista Formación Universitaria (en linea). volumen 9, número 1. La Serena-Chile (2016)

Dumas, M., M. La Rosa, J. Mendling y H. Reijers, Fundamentals of Business Process Management, Primera edición. Editorial Springer (2013)

Hallerbach, A., T. Bauer, y M. Reichert, Capturing variability in business process models: the Provop approach, Journal of Software Maintenance 22(6-7): 519-546 (2010)

Kumar, A. yW. Yao, Design and management of flexible process variants using templates and rules". Computers in Industry, Volume 63, Issue 2, February (2012)

La Rosa, M., M. Dumas, R. Uba y R. Dijkman, Business Process Model Merging: An Approach to Business Process Consolidation, ACM Transactions on Software Engineering and Methodology (2013)

Li, C., M. Reichert, y A. Wombacher, Mining business process variants: Challenges, scenarios, algorithms, Data \& Knowledge Engineering, Volume 70, Issue 5. (2011)

Llanera, M., S. Villodre, F. Pontoriero, A. Cattapan. "Modelo de gestión de la calidad para la puesta en marcha de cursos no presenciales: Instrumentos de seguimiento y evaluación, doi: 10.4067/S071850062014000600002. Revista Formación Universitaria (en linea). volumen 7, número 6. La Serena-Chile (2014)

Lohrmann M. y M. Reichert, Modeling business objectives for business process management, Proceedings SBPM ONE 2012 Scientific Research, LNBIP 104, Vienna, Springer, pp. 106-126 (2012)

Mattos, T., F. Santoro, K. Revoredo y V. Tavares, Formalizing the situation of a business process activity, Presentado en CSCWD 2012: 128-134 (2012)

Morrison, E., A. Menzies, G. Koliadis y A. K. Ghose, Business Process Integration: Method and Analysis, In Proc. Sixth Asia-Pacific Conference on Conceptual Modelling, APCCM (2009)

Ploesser, K., M. Peleg, P.Soffer, M.Rosemann, y J. Recker, Learning from Context to Improve Business Processes. BPTrends (2009)

Reichert, M.yA. Hallerbach y T. Bauer. Lifecycle Support for Business Process Variants. Springer, In: Handbook on Business Process Management, 2nd edition (2014)

Sanchis, R., R. Poler y A. Ortíz, Técnicas para el modelado de procesos de negocio en cadenas de suministro. Revista Información Tecnológica. volumen 20, número 2. La Serena-Chile (2009)

Schubert, P., y C. Legner, B2B integration in global supply chains: An identification of technical. Integration scenarios. Journal of Strategic Information Systems. (2011)

Tavares, V., C. Lima, y F. Santoro. Dynamic process adaptation: A context-aware approach. Presentado en CSCWD-11 (2011) 
